Slavica

bruxellensia

\section{Slavica bruxellensia}

Revue polyphonique de littérature, culture et histoire

slaves

9 | 2013

Érotisme

\title{
Traduction de poèmes de Miroslav Antić
}

Traduction du serbe

Jovana Slijepčević et Anthony Gaudillère

\section{CpenEdition}

1 Journals

Édition électronique

URL : http://journals.openedition.org/slavica/1322

DOI : $10.4000 /$ slavica.1322

ISSN : 2034-6395

Éditeur

Université libre de Bruxelles - ULB

Référence électronique

Jovana Slijepčević et Anthony Gaudillère, "Traduction de poèmes de Miroslav Antić », Slavica bruxellensia [En ligne], 9 | 2013, mis en ligne le 15 avril 2013, consulté le 10 décembre 2020. URL : http://journals.openedition.org/slavica/1322 ; DOI : https://doi.org/10.4000/slavica.1322

Ce document a été généré automatiquement le 10 décembre 2020.

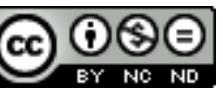

Les contenus de Slavica bruxellensia sont mis à disposition selon les termes de la Licence Creative Commons Attribution - Pas d'Utilisation Commerciale - Pas de Modification 3.0 France. 


\title{
Traduction de poèmes de Miroslav Antić
}

\author{
Traduction du serbe
}

Jovana Slijepčević et Anthony Gaudillère

\section{Présentation}

1 Décrire en quelques lignes la vie et l'œuvre de Miroslav Antić (1932-1986) semble relever d'une gageure, tant son activité fut incessante et se déploya dans de multiples directions, comme il le confessait lui-même :

J'ai fait n'importe quoi. J'ai été aide-maçon, ouvrier dans une brasserie, docker, marin, metteur en scène, je me suis occupé de plomberie et de canalisations, travaillé avec des compresseurs, façonné du bois, je sais construire un toit, j'ai joué dans un théâtre de marionnettes, et même fabriqué des marionnettes, animé des émissions à la télévision, été conférencier.

À cette liste il faudrait encore ajouter qu'il s'adonna à la peinture, réalisa des films et écrivit des livres pour enfants qui sont aujourd'hui dans tous les programmes scolaires serbes. Et dire qu'il fut aussi, et peut-être avant tout, un poète, un des plus grands qu'ait connus la poésie serbe dans la seconde moitié $\mathrm{du} \mathrm{XX}^{\mathrm{e}}$ siècle. Son talent fut d'ailleurs reconnu de son vivant : il reçut divers prix et distinctions pour ses activités en tant que journaliste, cinéaste et, donc, poète.

Antić commença à publier des poèmes dès l'âge de 16 ans. Parmi ses recueils les plus marquants, citons Plavo nebo (Le Ciel bleu, 1954), Psovke nežnosti (Les Jurons de la tendresse, 1959), Koncert za 1001 bubanj (Concert pour 1001 tambours, 1962), Mit o ptici (Le Mythe de l'oiseau, 1979), Savršenstvo vatre (La Perfection du feu, 1982). Ces recueils n'ont jusqu'à présent pas encore été traduits en français, tandis que la renommée d'Antić en tant que poète ne cesse de s'affirmer, comme en témoigne, entre autres, la tenue depuis 2008 à Novi Sad d'un festival culturel, "Antićevi dani » (Les journées d'Antić), à l'occasion duquel est attribué le prix «Miroslav Antić » qui récompense une création exceptionnelle dans le domaine de la littérature. 
Originaire de Voïvodine, Antić resta toute sa vie intimement attaché à sa région natale. À l'image de cette terre cosmopolite, à la croisée des mondes, sa poésie est nourrie de nombreuses influences et porte en elle une gamme composite de teintes et de tonalités. Si le je est omniprésent dans ses poèmes, c'est pour mieux aller à la rencontre du monde dans ce qu'il a d'inattendu et d'invraisemblable. Antić évite tout pathos, se détourne des lieux communs, et convoque pour cela une langue où se heurtent des mots qui n'auraient pas dû se côtoyer, donnant à chacun de ses poèmes une dynamique intérieure propre où règne une atmosphère souvent onirique et irréelle. De la même manière, sa poésie aime à mêler les formes, en un procédé qui n'est pas sans rappeler certains poètes russes qu'il appréciait, tels que Vladimir Vl. Majakovskij ou Sergej A. Esenin ; ainsi ses poèmes se font tour à tour, et parfois en même temps, prières, jurons, hymnes, confessions, récits.

Antić est aussi le poète des quatre éléments. Il y a l'eau: l'eau des larmes et des crachats, l'eau des mers et des rivières que l'ancien marin n'a pas oubliées et dont on trouve des réminiscences dans ses vers. Il y a le feu, symbole de perfection auquel il dédia tout un recueil. Il y a l'air, et ces vents par lesquels le poète rêve de se faire happer, à l'instar de l'oiseau, animal de liberté suprême dont l'image revient de façon obsessive dans son œuvre. Et il y a la terre, à laquelle les hommes, de par leur condition de mortel, restent tragiquement attachés, et à laquelle le poète, lui, tente d'échapper.

Il y a ainsi chez Antić comme un impératif, celui de rester vivant, coûte que coûte. Pour cela, il lui faut défier la raison, célébrer l'amour et braver la mort. Défier la raison, en invoquant une métaphysique qui bouscule les règles les plus élémentaires de la logique. Célébrer l'amour, cette mesure d'innocence et de souffrance sans cesse recommencées. Braver la mort, enfin, en la regardant dans les yeux, en visitant sa propre tombe ou en écrivant une épitaphe qui n'en est pas vraiment une, comme pour se rendre immortel.

Et si la mort malgré tout advenait, Antić avait pris soin d'adresser à ses lecteurs le vœu suivant :

Ce que je préférerais, c'est que vous inventiez ma biographie vous-mêmes. J'aurai alors beaucoup de vies diverses et je serai le plus vivant parmi les vivants.

Le reste, ce qu'il ne faut pas dévoiler d'un écrivain, mais plutôt le chuchoter, je l'ai dit dans mon poème In Memoriam.

Et dans tous mes autres poèmes.

\section{Traduction}

\section{In Memoriam}

Il y a un incroyable salaud qui s'appelle Miroslav Antić.

Il bouffe mon pain. Fait mes enfants. Porte mes habits.

Il couche dans mon lit avec ma femme devant mes propres yeux,

Car il sait qu'en ce moment je dois être quelque part loin à Léningrad.

Et cet Antić qui m'a détruit et comme écrivain et comme homme,

Celui-là donc, qui en fin de compte ira se coucher dans ma propre tombe,

Me demande un matin: qu'est-ce qui vous arrive, vieux, vous m'avez l'air un peu malade?

Pardonnez l'expression, mais qu'est-ce que ça peut lui foutre, comment je vais et où j'en 
suis?

Ceux qui s'occupent le mieux de moi sont ceux qui me laissent en paix.

Et lui se lave les mains avec ma rakia. Il a les clefs de mon atelier.

Voyez donc, celui-là me force à lire des livres. Il manigance avec mes blondes.

Il hurle dans ma maison. Me calomnie. Raconte n'importe quoi.

Mes enfants lui ressemblent, et lui porte une cravate.

Il se rase, connaît des gens. Il travaille.

Chaque matin il se douche. Il fait celui qui sait tout sur le bouddhisme zen.

Il traduit des livres. Croit qu'il a des amis.

A mon fils, imaginez cette saloperie, à mon fils unique,

Il achète des glaces.

J'étais marin. Je fuyais ou, tenez, une fois, je pars pour Paris.

Je mets la couverture sur ma tête. Me laisse pousser la moustache.

Et même là il me retrouve, dans une rue Jolivet, dans un hôtel miteux,

Et me ramène à la maison. Et me fait pleurer.

Ma mère Mélanie, qui ne sait pas qu'elle m'a mis au monde moi et non pas lui, Elle l'aime plus. Le croit plus. Et comme il en profite.

Mais lui, je vous jure, il est cette bête endimanchée à qui je dresse un monument.

Il est cet animal estimé à qui j'écris une biographie

Ainsi conspué et seul, et totalement dégoûté

De devoir publiquement lui prêter mes yeux et mon âme

Et le peu d'argent que j'ai emprunté avec peine.

Moi par exemple quand j'ai sauté de la forteresse de Petrovaradin

Lui plongeait dans les manuels scolaires.

Quand le docteur Savić me soignait de l'alcool,

Il faisait celui qui s'y connaît en cinéma.

Où que je sois, il m'obligeait à ne pas lui faire honte.

Il consentait aux compromis. Ricanait aux réceptions.

Recevait mes prix. S'immisçait dans mes rêves.

Un hypocrite.

Un vrai hypocrite.

Un provincial!

Un homme qui a supporté tout ce que je ne supporterai jamais.

Et qui maintenant se presse bien de crever à ma place,

Pour qu'à ma place,

Ce porc,

Pour qu'à ma place bien vite lui seul vive. 


\section{Paris}

1.

Un homme s'en va dans la rue Montparnasse, pour acheter dans le magasin du coin, chez Inno, des espadrilles en solde, et d'un coup se rend compte qu'il est mort. Des gens lui demandent quelque chose, et lui leur répond. Pourquoi pas? Et le plus merveilleux, c'est qu'il ne panique pas du tout.

2.

Une blonde, avec qui il a rendez-vous à cinq heures et demie, attend à la sortie du métro Edgar Quinet. L'homme lui dit: allons-y. Puis ils partent pour sa tombe, 2 rue Jolivet.

3.

La femme prend un long bain et se sert de trois sortes de déodorants. Et après ça, d'un coup, elle ne veut plus sortir de sa vie, parce que les femmes remarquent tardivement celui qui est mort pour de vrai.

4.

L'homme sourit. Il lui récite en tchèque : Maryčka Magdonova de Petr Bezruč. Quand le vieux Magdon de la chasse revenait à la maison... et cætera. Dans ses nouvelles espadrilles et propre comme pour un enterrement, il lui prend les cheveux et en fait une couronne qu'il se pose sur le ventre. Elle appelle cela : le charme slave.

5.

Ils se séparent devant le métro. Il dit: les maisons, qui n'ont jamais eu le besoin particulier de grimper dans les hauteurs, et le ciel, qui a souvent dû pencher la tête et descendre jusqu'à ces toits, c'est ça Paris, non? Elle lui embrasse la paume de la main et dit : je réfléchirai.

6.

L'homme est de nouveau seul et s'habitue petit à petit à sa nouvelle mort, à peine entamée. Au lieu de traverser la rue jusqu'au cimetière, à même pas dix pas, et de se coucher, discrètement, sans faire de vacarme, dans une des meilleures tombes, il prolonge vers Bobino.

7.

Et là-bas, dans sa déjà trop grande mort, il meurt encore plus, en murmurant avec Léo Ferré quelque refrain d'un autre mort, Boris Vian, quatre vers sur la vie, qui n'ont maintenant plus aucun sens.

\section{Belgrade}

1.

Je ne veux pas étudier le droit, car je ne pourrais jamais prouver au monde qu'il était juste que tu aies jeté sous le train ton enfant blanc ce printemps-là. 
2.

Ni la médecine, car je ne pourrais jamais soigner les petites fraises souffrantes qui ont mûri sur ton ventre jadis si ferme.

3.

Et les poèmes je les écrirai toujours aussi sincères et hideux, semblables à cette réfection enfumée, à notre nouvelle rencontre de ce soir, dix ans après.

4.

Nous étions tous amoureux de toi dans le quartier. Une ruée de jouvenceaux. Comment m'aurais-tu reconnu dans cette cohue? Que ton pied lutte avec le mien sous la table, car nous tous, finalement, dans ce monde, luttons comme nous le pouvons.

5.

Après toi il restera les mêmes rues, et les enfants qui apprennent à l'école à dessiner le printemps, et les livreurs de journaux, mais personne ne te pleurera, personne ne hurlera à la lune comme un fou, bien que tu aies porté dans tes yeux gris un monde qui valait plus que toutes les guerres, que les centrales hydrauliques, que les gains en bourse et à la roulette.

6.

Et j'aimerais tant, moi qui détestais tous les rois, que tu deviennes cette nuit une reine, qu'on te porte dans les places de la ville, qu'on s'incline devant toi, qu'on t'acclame et qu'on écrive des vers sur tes yeux et tes cheveux.

7.

On obligerait et les feuilles, et les ballerines de l'opéra national, et les manèges de foire, et les publicités lumineuses à tourner en rond comme des millions de poussières dans ton sang.

8.

Prends tes bagages. Le train part une minute après minuit. Je ne suis ni l'Ange Gabriel, ni un policier qui s'occupe des voyageurs. Je suis juste un poète ivre et je saluerai longtemps le train, car nous sommes toi et moi de la même famille : celle de l'humanité.

\section{Concert pour 1001 tambours}

\section{Prologue (I-V)}

I

Egorgez donc enfin vos violons

Et essuyez les archets sur vos manches.

Mettez-vous sur la pointe des pieds quand on vous baptise.

Et quand on embrasse votre front. 
Et quand on crache sur vos yeux bleus.

Eteignez les chandelles.

Et les fleurs.

Et les herbes.

Les étés d'innocence ont passé.

En cette nuit, froissée comme une couverture

Entre les jambes de religieuses

Qui souffrent d'insomnies,

Je suis le dernier ciel bon marché

Pour tous les rêveurs

Et tous les oiseaux ivres.

Prêtez l'oreille, les étoiles aux rudes crêtes Coqueriquent le vent d'automne.

II

Peut-être est-ce la vieillesse qui a effleuré

Nos lèvres

Et nos joues pâles,

Et nous a visés à travers les prunelles

Avec deux fusils défunts.

Pourquoi,

Sinon,

Le silence somnolent étreindrait-il la rangée d'arbres,

Comme un soldat grisé les cuisses refroidies d'une servante

Lors des adieux

Dans le parc

Derrière le stade de football ?

Madame la ville,

Vous ne méritez pas les infinis défilés nocturnes des oiseaux

Au-dessus des toits depuis longtemps gris,

Ni les larmes jaunes dans les yeux des poteaux,

Le long de toutes ces rues,

Où l'on a vécu notre unique jeunesse en bâillant.

Madame la ville, assez des parades,

Des feux d'artifice et des cloches.

III

Je ne me suis sans doute pas imaginé que les arbres

Sous mes fenêtres sanglotent.

Et jouent

A l'orgue, 
Malgré leurs mains si écorchées,

Si fanées,

Si encroûtées?

En cette nuit,

Quand tous impassiblement marchent au pas vers le cimetière,

Marchent au pas,

Et traînent dans la boue leurs couronnes de fleurs en lambeaux

Et leurs drapeaux rapiécés,

Sur le plus grand champ de bataille de l'amour et de la honte

Je lève de la main les couches tricolores

De mon fils à naître.

En cette nuit, quand le brouillard au loin

Se fige et se répand,

Quand personne n'essaye même

De comprendre ces étincelles dans les prunelles de mes yeux,

Je ne me suis pas imaginé, sans doute,

Que je suis le seul, ainsi étoilé, qui soit fou

Et naif,

Et splendide,

Splendide comme la vitrine d'un antiquaire de banlieue

Dans laquelle il y a des mines.

IV

Vent,

Je vous en prie,

Jetez les feuilles dans la rivière

Comme les enfants jettent leurs ballons de baudruche éclatés.

L'automne est la plus bête tristesse parmi les tristesses.

L'automne est mon amour

Aux cinq lettres aux yeux verts,

Et le mensonge,

Le plus enfantin mensonge

Grâce auquel je suis mauvais et bon jusqu'à en perdre conscience.

Vent,

Qu'est-ce qu'on sait nous deux,

Peut-être, en ce moment, dans tous les wagons jaunes,

Sur de lointaines voies ferrées jaunes,

Entrent les petites filles de nos rêves jaunes,

Des petites filles aux cheveux jaunes comme le blé.

Pourquoi donc, 
Sinon,

Tout cela porterait-il un nom si jaune : octobre?

V

Nous, qui balbutions ainsi d'une voix enrouée

Et qui embrassons,

Nous, brossés soigneusement comme de précieux habits,

Et séchés dans les souvenirs des autres

Comme des carcasses ayant fait leur temps,

Nous avons en cette nuit mérité cette dernière navigation,

La plus belle d'entre toutes,

N'est-ce pas, vent?

Nous l'avons méritée en cette nuit, quand sous la pluie pourrit le monde.

Son nom blanc entre nos dents engourdis

A le goût du sang coulant et de la mûre.

Quand nous l'aimons, c'est si terriblement rouge,

Rouge jusqu'au fond de notre foie.

Quand nous l'aimons,

C'est comme quand nous nous précipitons

Le ventre nu sur une baïonnette.

Epilogue (LII-LIII)

LII

Ceci n'est pas une confession.

Ceci est pire qu'une prière.

Mille fois depuis ce matin

Je t'aime comme jadis.

Mille fois depuis ce matin

Je te reviens de nouveau.

Mille fois depuis ce matin

J'ai de nouveau peur

Pour toi,

Perdue dans le tourbillon des cartes géographiques,

Pour toi,

Distribuée comme une affiche

A on ne sait qui.

Est-ce que je suis encore cette mesure

Selon laquelle tu sais de qui tu souffres? 
Selon laquelle tu sais combien les autres

Ont été nus devant toi?

Cette mesure selon laquelle tu sais qui te dérobe

Et qui paye?

Est-ce que je suis toujours

Parmi toutes tes vies

Ce petit bout du plus pur nuage dans ta poitrine,

Et du plus sanglant nid d'abeilles?

LIII

Tu es la seule qui ait nourri toute ma faim

Avec ce peu de viande

Et de rêve.

La seule rassasiée jusqu'aux dents

De ce peu de mes ongles

Et de mes paumes.

J'aimerais que tes futurs fils

Héritent du timbre de ma voix

Et que tes filles portent ma tristesse

Dans leurs petits gilets de soie.

J'aimerais toutefois que tu conserves quelque part Toutes mes lointaines cimes

A l'horizontale de ton abîme.

Et que tu fasses passer mes yeux trop mûrs

A travers le silence des yeux de tous les autres

Et celui de leurs appartements.

Et mon octobre à travers tous les avrils des autres.

\section{La Perfection du feu}

\section{I, 8}

Depuis des jours j'épie les oiseaux. D'eux j'apprends à voler. J'apprends comment être un cousin du vent.

Peu à peu je perds le passé. J'arrête de me souvenir D'où je suis venu.

En jouant à fendre un espace d'un autre, Je ne saurai bientôt plus si je suis tout à fait là. 
Ces systèmes étourdis sont comme l'homme,

Ils alertent très sérieusement.

Le temps est venu, me semble-t-il,

De me mettre en vol,

Et de toucher des lèvres

La pellicule entre la fin et le début.

I, 30

Ma durée ne s'accorde pas à la vôtre.

J'en ai outre mesure. Vous en manquez par trop.

Mon œuvre précède les pensées.

La vôtre les accompagne.

La somme de mes lieux n'est pas la somme de vos lieux.

Les points condensés comme celui-ci sont rares.

On les appelle : économie du temps

Et infini de la vitesse. C'est ici que nous nous rencontrons.

Ce n'est qu'ainsi que l'absence de forme s'installe dans les formes de la conscience,

Et qu'on reconnaît les couches des espaces nouveaux et inattendus.

Facile d'être différents parmi les mêmes.

Difficile de se distinguer de soi-même.

IV, 92

Comme un oiseau, qui essaye de voler à l'envers.

Et comme une pierre, qui s'évertue à tomber vers le haut.

Comme une mer, qui croit secrètement qu'elle débordera

Dans ses affluents. Ou une cendre, qui tente

Par tous les moyens de devenir fournaise,

Ou du moins le bout d'une flamme, n'importe quelle chaleur.

Comme un poisson, qui veut redevenir rogue.

Comme un os d'éléphant, des métaux, une nacre ou un arbre,

Qui veulent redevenir des êtres. Comme une séparation

Qui s'imagine être une rencontre. Voilà, ainsi

L'avenir entre-t-il en moi, en ne pressentant pas que j'ai, Juste à l'instant, survécu à tous ses lointains descendants. 


\section{BIBLIOGRAPHIE}

Antić M., Savršenstvo vatre (La Perfection du feu), Prometej, Novi Sad, 1997 ;

Antić M., Koncert za 1001 bubanj (Concert pour 1001 tambours), Prometej, Novi Sad, 1998 ;

Antić M., Nama svima od Mike Antića - izbor i predgovor Jovo Miladić (À nous tous de Mika Antić choix et préface de Jovo Miladić), Prosveta, Beograd, 2007.

INDEX

Mots-clés : littérature serbe

Index géographique : Serbie, Yougoslavie

Index chronologique : communisme, époque contemporaine, XXe siècle

\section{AUTEURS}

JOVANA SLIJEPČEVIĆ

Professeur de français à l'Institut français du Monténégro

\section{ANTHONY GAUDILLÈRE}

Professeur de français à l'Institut français du Monténégro 